\title{
Teledermatology in the Saudi Arabian Healthcare System: Utilization and Challenges
}

\author{
Alhanouf A. Bin Dakhil ${ }^{\mathrm{a}}$ Saad Altalhab ${ }^{\mathrm{b}}$ \\ ${ }^{a}$ College of Medicine, Al-Imam Mohammad Ibn Saud Islamic University, Riyadh, Saudi Arabia; ${ }^{b}$ Department of \\ Dermatology, College of Medicine, Al-Imam Mohammad Ibn Saud Islamic University, Riyadh, Saudi Arabia
}

\section{Keywords}

Teledermatology $\cdot$ Telehealth · Skin diseases · Healthcare · Telemedicine

\begin{abstract}
Background: In recent years, telemedicine has led to a dramatic shift in healthcare service delivery, mainly due to the ease with which telemedicine can be integrated into a multitude of specialties and its flexibility as a means of providing care. Over the last 2 decades, technological advancements have made telemedicine integral to healthcare in many countries. In particular, dermatology was benefited from telemedicine as a new tool, thanks to the visual character that pervades this practice. Teledermatology is the interactive practice of remote dermatological assessment, involving live contact with patients ("live interactive") and data access and retrieval ("store-and-forward"). This review discusses the role of this new discipline in medical education, inpatient care, and primary care through the analysis of several studies addressing this topic. Also examined are the status and limitations of teledermatology in Saudi Arabia and some proposed solutions. Summary: Visual assessment of dermatological conditions is common practice among dermatologists. However, due to the scarcity of specialists in
\end{abstract}

karger@karger.com www.karger.com/sjh

Karger!"

GOPEN ACCESS
(C) 2021 The Author(s)

Published by S. Karger AG, Basel

This is an Open Access article licensed under the Creative Commons Attribution-NonCommercial-4.0 International License (CC BY-NC) (http://www.karger.com/Services/OpenAccessLicense), applicable to the online version of the article only. Usage and distribution for commercial purposes requires written permission. some areas, wait times have been a hindrance for patients requiring an assessment. Teledermatology improves access by reducing wait times, speeding up the referral process, and streamlining assessments. Furthermore, teledermatology effectively serves as a form of triage, particularly for cases of suspected cutaneous malignancy that typically require the shortest referral time. Earlier diagnoses, more effective management of chronic skin disease, and inpatient care are all additional benefits offered by the teledermatology practice. The use of teledermatology is now widespread, with roles extending beyond patient care to medical teaching and training in residency programs. This perhaps reflects physicians' highly positive perceptions regarding teledermatology; however, patient perception in Saudi Arabia is still lagging behind the global standard, possibly due to privacy concerns. Key Message: Teledermatology can be reliably utilized to advance healthcare services and medical education. Improving patient awareness and perception of this emerging discipline is crucial; to that end, the practice must address privacy concerns. Patient uploads of photographs and videos should be stored on an end-to-end encrypted platform to provide optimal service and encourage patients' participation. Ultimately, physicians should be well acquainted with the medical, ethical, and technical aspects of teledermatology.

(c) 2021 The Author(s)

Published by S. Karger AG, Basel 


\section{Introduction}

Overall, telemedicine is heavily dependent on the technology and communications industries to allow remote access to healthcare professionals for patients, regardless of their physical location [1]. It can be broadly defined as "the delivery of healthcare services, where distance is a critical factor, by all healthcare professionals using information and communication technologies for the exchange of valid information for the diagnosis, treatment and prevention of disease and injuries, research and evaluation, and the continuing education of healthcare providers, all in the interests of advancing the health of individuals and their communities" [2]. The field of telemedicine includes utilizing ongoing remote support with healthcare professionals to address and manage an individual's medical care. This involves direct communication and other digital mediation techniques [3]. For instance, consultations and follow-up visits can be scheduled and carried out online. In the former case, this is known as an e-consultation [1].

When telemedicine is used to practice dermatology, the discipline is referred to as teledermatology [4]. This is further divided into 2 classes: real-time and store-andforward teledermatology [4]. Real-time teledermatology is a form of live telemedicine, with a patient connecting to a dermatologist by means of digital communication such as a video chat. In store-and-forward teledermatology, either the healthcare provider or patient sends images or videos to a teleconsultant, who then investigates the media and responds with a relevant diagnosis or management plan [4]. When elements of both these methods are present, it is referred to as "hybrid teledermatology." The last form of this care is mobile teledermatology, which describes the practice of teledermatology using mobile phones [4].

The technological advancement of recent decades has been a boon to telemedicine around the world, and various innovations are rapidly being integrated into modern healthcare services [5]. Teledermatology has been a core part of that development, largely thanks to the visual nature of dermatological assessment and consultation [5]. Healthcare systems have been improved by the use of teledermatology because it allows patients to gain faster access to dermatologists. This practice is effective in shortening wait times and lessening the need for in-person visits, thereby relieving pressure on overburdened healthcare systems [6]. It also provides specialist healthcare that some patients might otherwise struggle to access (e.g., patients in remote areas).
This article discusses the state of teledermatology, particularly in Saudi Arabia. A logical combination of the terms "Saudi Arabia," "telehealth," "telemedicine," "teledermatology," and/or "education" was used to search PubMed, Scopus, and Google Scholar databases. We analyzed both local and international studies to survey teledermatology's use and reliability for disease management and diagnosis relative to face-to-face healthcare. Primary care, inpatient, and medical education settings of teledermatology are reviewed. Moreover, limitations and challenges to the practice of teledermatology in Saudi Arabia are discussed, followed by the proposal of specific solutions and recommendations.

\section{Teledermatology's Reliability and Usefulness in Primary Healthcare}

Within the realm of primary care, teledermatology can be used effectively in triage and management with respect to skin conditions [6]. Primary care physicians can use teledermatology to consult with dermatologists in a prompt manner. This allows primary care physicians to assess, diagnose, and treat common skin conditions that would otherwise be holding up the line. It can also be used for follow-up appointments in the case of chronic skin conditions, such as chronic ulcers [4]. This helps reduce the need for face-to-face appointments with dermatologists for common skin diseases and conditions, which allows in-person visits to become more available to those with serious or severe disorders.

There are many examples of teledermatology being used effectively and reliably as a consultation framework, with Trettel et al. [5] reporting 138 studies that found teleconsultation feasible, reliable, and/or effective. Of these, $n=34$ were reported to be superior to face-to-face settings, $n=25$ equivalent, and $n=15$ inferior [5]. A study by McAfee et al. [7] involved the evaluation of 25 Cleveland Clinic Health System walk-in primary care clinics. The researchers investigated a teledermatology e-consultation program that utilized a store-and-forward approach, through which $500 \mathrm{pa}$ tients received consultation over 517 e-consultations [7]. The median time elapsed from placement in the e-consultation system to completion of consultation was just 1 day [7]. In $71 \%$ of cases, "teledermatologists" were able to find discordant results to primary care physicians, and in $60 \%$ of cases, a change in the treatment plan was recommended [7]. This measurement demonstrates the utility of such a program for both walk-in and primary care clinics [7]. Patients were granted access to board-certified dermatology professionals who, in strictly in-person circumstances, 
would likely have waited much longer to see. This saved patients' time and expenses and, in some cases, positively affected their health outcomes [7].

Moreover, Giavina-Bianchi et al. [6] carried out a cross-sectional study on individuals $(n=30,976)$ with skin lesions that were evaluated via teledermatology consultations. Wait times, frequency of diagnoses, and frequency of referrals to in-person specialists for biopsies were compared against standard dermatological consultation [6]. And, 53\% of cases were managed effectively by their primary care physicians, $43 \%$ went to referral for an in-person consultation, and $4 \%$ were referred directly to biopsy. The result was a $78 \%$ reduction in wait times for in-person appointments [6].

\section{Teledermatology in Inpatient Services}

Traditionally, teledermatology has been primarily implemented in outpatient care. However, recent studies are demonstrating its effectiveness for inpatient dermatology as well [4]. Because inpatient access to dermatologists is typically limited, teledermatology represents a novel solution to this long-standing accessibility problem [8]. When dermatologists perform inpatient consults, the patient outcomes are largely improved; similarly, inpatient teledermatology functions to reduce the wait time for a patient to receive such care [8] (14). There is considerable interest in inpatient teledermatology among surveyed dermatologists [8]. Weig et al. [9] found that $61.5 \%$ of dermatologists either agreed or strongly agreed with the statement that teledermatology was helpful for inpatient care. In a separate study, 95\% of hospitals and emergency departments were found to answer in the affirmative when asked if they would utilize a teledermatology consult service were it offered [10].

Another study by Gabel et al. [8] involving 27 dermatologists and 41 patients assessed the diagnosis and management effectiveness of teledermatology consultations. Substantial agreement was found between teledermatology assessment results and in-person dermatological results for diagnosis and laboratory workup decisions, with an almost perfect agreement regarding imaging [8].

\section{Teledermatology in Medical Education}

There are a number of additional educational benefits to teledermatology for both primary care providers and dermatologists [4]. The expanded knowledge and public health policies that teledermatology facilitates through increased collaboration between primary care providers and dermatologists will result in the advancement of both the prevention of the most common skin diseases, as well as increased training for their treatment and management [6]. Teledermatology may also play a significant role in resident physician training, further improving education outcomes.

While many dermatology residencies now have established teledermatology programs, relatively few have assessed their impact, with no such assessment having been conducted in Saudi Arabia. Resident perspectives on teledermatology were evaluated by Zakaria et al. [11] with an aim to quantify case evaluation numbers that result from such programs. Their results show that although teledermatology provides residents with a higher caseload, they may carry out their job in a lower stress environment. This encourages the comprehensive clinical assessment and consideration of broad and differential diagnoses, thus fostering visual, diagnostic, and triage skills [11]. Productivity rations (patients per hour) were 11.49 and 4.55 for teledermatology sessions and in-person clinic sessions, respectively [11]. Residents therefore assessed more than twice the number of patients at the same time, implying that the process would be highly effective in developing the abilities of residents in training [11].

\section{Teledermatology in Saudi Arabia}

The number of officially documented dermatologists in the national healthcare system in Saudi Arabia is limited [12]. The rate of dermatologists per population in Saudi Arabia is 1 per 15,000, with the number of dermatologists being scarcely over 2,000 , and $<30 \%$ of the registered dermatologists working in government hospitals [12, 13]. Thus, the utilization of teledermatology in this particular part of the world is crucial, especially in the government sector. European and North American healthcare systems have the highest rate of teledermatology implementation. Meanwhile, countries with less uniform geographical distributions of physicians are poorly represented in teledermatology practice - a concept revealed through a systematic review administered by Trettel et al. [5]. While these particular studies and those measuring effectiveness or satisfaction are lacking in the Saudi Arabian context, Alakeel [14] found that telemedicine overall is witnessing high levels of satisfaction and participation among dermatologists in the kingdom, with $>50 \%$ of study participants indicating they had actively engaged in a telemedicine session at least once. Reasons given for lack of active participation included technological limitations, insufficient infrastructure, 
and unreliable software tools, with very few giving a poor perception of telemedicine as a reason [14].

Generally, telemedicine is more closely studied in Saudi Arabia than teledermatology specifically. In one study, Saudi Arabian physicians from 4 tertiary clinics were surveyed to assess knowledge and perceptions of telemedicine, with $90 \%$ reporting it as a feasible method for providing medical care [1]. Nonetheless, >three-quarters (77\%) also reported that regular training would be necessary for the care to be adequate through such channels [1]. A separate study by Kaliyadan et al. [15] found that during the COVID-19 pandemic, 58\% of Saudi Arabian clinicians utilized telemedicine at least once.

Patient perception of telemedicine in Saudi Arabia is not quite as positive. $81 \%$ of patients in a study by $\mathrm{Al}$ Kuwaiti et al. [16] thought that better care is provided when physicians access and use electronic records and tools; however, of those surveyed, a full $65 \%$ still preferred in-person visits as opposed to virtual. In another study, Al-Anezi [17] found an even more negative view among Saudi Arabians, with a significant majority of respondents (84\%), indicating that they did not have trust in telemedicine services. A small proportion $(4 \%)$ reported having confidence in medications prescribed by a physician who had not performed the physical examination in person [17]. However, this study was not particular to any subfield of telemedicine; that is, teledermatology was not mentioned. It is, therefore, unclear whether such patients would have a different view for specific fields in which visual examination is the primary means of assessment, such as teledermatology. Thus, the previous results may not be meaningfully applied to patient perception of teledermatology. Furthermore, whether or not patients were concerned with their privacy or the security of their personal health documents was not assessed. The aforementioned study by Kaliyadan et al. [15] found that, interestingly, a majority of clinicians were using WhatsApp ${ }^{\circledR}(211,53.8 \%)$ and Zoom ${ }^{\circledR}(131,33.4 \%)$ for their telemedicine services, with only $16.5 \%$ using Sehha ${ }^{\circledR}$, the application specifically designed for remote healthcare by the Ministry of Health. No studies have been performed to measure which among these (or other) platforms inspire higher trust or confidence in the patients using them.

\section{Limitations and Strategies to Improve Teledermatology in Saudi Arabia}

In a broad sense, telemedicine is accompanied by several limitations, such as the lack of ability to perform certain elements of a physical examination. Despite this, the most visual nature of the field of dermatology makes teledermatology one of the least affected forms of telemedicine in regard to this disadvantage. However, in cases of potentially malignant lesions, teledermatology does not eliminate the need for an in-person visit to address such conditions through physical examination and biopsy procedures. Moreover, telemedicine requires that healthcare providers be intimately familiar with the use of technological communication. Due to the requirements for technological infrastructure and reliable internet connectivity on both sides of an e-consultation, teledermatology is not necessarily feasible in all geographical areas [4]. The absence of an appropriate supporting infrastructure would lessen or eliminate the effectiveness of teledermatology [4]. However, as technology continues to improve, both in terms of its quality (e.g., clarity of smartphone photographs) and accessibility (e.g., expanding reach of connectivity), outcomes within this field should see improvement [4]. Recent studies show that patients are able to take accurate and high-quality dermatological photographs from their own homes, although in some areas, such as the oral cavity, it may still prove difficult [4]. Such photographs are particularly important for the assessment and management of potentially malignant lesions and other dermatological conditions affecting the oral cavity.

Three oral medicine centers in the USA assessed their patient and provider satisfaction in oral telemedicine through a cross-sectional study. While patients were found to be satisfied with the services they received, most utilized e-consultations to avoid long travel distances. Among 108 oral telemedicine visits, $42 \%$ of physicians disagreed that the oral examination was easy to perform [18]. To improve the quality of teledermatology outcomes, standard protocols for imaging and other teaching materials should be provided to patients to allow for a smoother experience [4].

Electronic medical records, while common practice across the developed healthcare systems, differ greatly in the extent and manner in which they are used [4]. The effectiveness and capabilities of teledermatology are significantly impacted by such records, requiring an adequate system for storing and retrieving records involving patients' images [4]. Such electronic health record platforms must be secure, reliable, and adaptable in order to be implemented into a variety of regulatory, medical, and professional compliance contexts around the globe [1]. Secure, medically oriented platforms may improve patient sentiment and decrease hesitancy regarding telemedicine compared to some of the currently used platforms that are tailored to social communication. 
All teledermatology practitioners must have at least a basic understanding of dermatological imaging and nomenclature in order to practice [4]. National legal frameworks must also be taken into account, since they may differ from those related to traditional in-person services [4]. The indefinite security of patient health information should be of critical importance at all times to the teledermatology practitioner. Finally, teledermatology business models must take into account reimbursement matters, particularly with ongoing transformations occurring within the Saudi Arabian healthcare system.

\section{Conclusion}

Wait times, appropriate specialists' referrals, and triage are all factors that can be notably improved through the use of teledermatology. Early diagnosis and treatment of chronic skin diseases will also see improvement with the use of this tool. Additionally, for patients receiving aesthetic treatments - particularly follow-up care - teledermatology can be quite effective (e.g., monitoring laser resurfacing patients post-procedure) [4]. Furthermore, interpretation of patch tests has been successful through teledermatology [4]. Teledermatology can also play an important role during inpatient care, improving patient access and lowering wait times. Moreover, teledermatology has been effectively utilized in educational settings, such as residency programs [4]. The field provides a highly costeffective method for mobilizing expert faculty into teaching programs almost anywhere [4]. Strategies should be adopted to improve patient perception of telemedicine and teledermatology as its public opinion varies considerably and, in some cases, can even be negative. Patient awareness of the reliability and effectiveness of teledermatology tools is critical. Finally, the use of encrypted devices and platforms to share and store patient images is essential to conducting appropriate teledermatology services.

\section{Conflict of Interest Statement}

Dr. Bin Dakhil has no conflicts of interest to declare. Dr. Altalhab has no conflicts of interest to declare.

\section{Funding Sources}

No funding was reported.

\section{Author Contributions}

Alhanouf A. Bin Dakhil: conceptualization, design, writing, editing, and literature review. Saad Altalhab: edited and supervised the manuscript.

\section{References}

1 Almathami HKY, Win KT, Vlahu-Gjorgievska E. Barriers and facilitators that influence telemedicine-based, real-time, online consultation at patients' homes: systematic literature review. J Med Internet Res. 2020;22(2):e16407.

2 World Health Organization. Telemedicine: opportunities and developments in member states. Report on the second global survey on eHealth: World Health Organization; 2010.

3 Blandford A, Wesson J, Amalberti R, AlHazme R, Allwihan R. Opportunities and challenges for telehealth within, and beyond, a pandemic. Lancet Glob Health. 2020;8(11):e1364-5.

4 Kaliyadan F, Ramsey ML. Teledermatology. StatPearls. Treasure Island, FL: StatPearls Publishing Copyright $\odot$ 2021, StatPearls Publishing LLC.; 2021.

5 Trettel A, Eissing L, Augustin M. Telemedicine in dermatology: findings and experiences worldwide: a systematic literature review. J Eur Acad Dermatol Venereol. 2018;32(2):215-24.

6 Giavina-Bianchi M, Santos AP, Cordioli E. Teledermatology reduces dermatology referrals and improves access to specialists. EClinicalMedicine. 2020;29-30:100641.
7 McAfee JL, Vij A, Warren CB. Store-and-forward teledermatology improves care and reduces dermatology referrals from walk-in clinics: a Retrospective Descriptive Study. J Am Acad Dermatol. 2020;82(2):499-501.

8 Gabel CK, Nguyen E, Karmouta R, Liu KJ, Zhou G, Alloo A, et al. Use of teledermatology by dermatology hospitalists is effective in the diagnosis and management of inpatient disease. J Am Acad Dermatol. 2021 Jun;84(6):154753.

9 Weig EA, Tull R, Chung J, Wanat KA. Inpatient teledermatology: current state and practice gaps. J Am Acad Dermatol. 2020;83(3):797-802.

10 Cheeley J, Chen S, Swerlick R. Consultative teledermatology in the emergency department and inpatient wards: a survey of potential referring providers. J Am Acad Dermatol. 2018; 79(2):384-6.

11 Zakaria A, Maurer T, Amerson E. Impact of teledermatology program on dermatology resident experience and education. Telemed J E Health. 2020.

12 Bindakhil M, Charmelo-Silva S, Bin Dakhil AA, Alomair IA. The value of the oral medicine specialty in the modern healthcare systems. Saudi J Health Sys Res. 2021:3340.
13 Ministry of Health, Kingdom of Saudi Arabia Statistical Yearbook 1440H. 2020 [cited 2021 July 25]. Available from: https://www.moh.gov. sa/en/Ministry/Statistics/book/Pages/default. aspx.

14 Alakeel A. Acceptance of teledermatological practices: a Cross-Sectional Study of Practicing Saudi Dermatologists. Cureus. 2021;13(3): e13710.

15 Kaliyadan F, A Al Ameer M, Al Ameer A, Al Alwan Q. Telemedicine practice in Saudi Arabia during the COVID-19 pandemic. Cureus. 2020;12(12):e12004.

16 Al Kuwaiti A, Al Muhanna FA, Al Amri S. Implementation of digital health technology at academic medical centers in Saudi Arabia. Oman Med J. 2018;33(5):367-73.

$17 \mathrm{Al}$-Anezi FM. Evaluating the readiness of mobile technology with respect to e-Heath for medication in Saudi Arabia: an integrative perspective. J Multidiscip Healthc. 2021;14:59-66.

18 Villa A, Sankar V, Shazib MA, Ramos D, Veluppillai $\mathrm{P}, \mathrm{Wu} \mathrm{A}$, et al. Patient and providers' satisfaction with tele(oral)medicine during the COVID-19 pandemic. Oral Dis. 2020. 